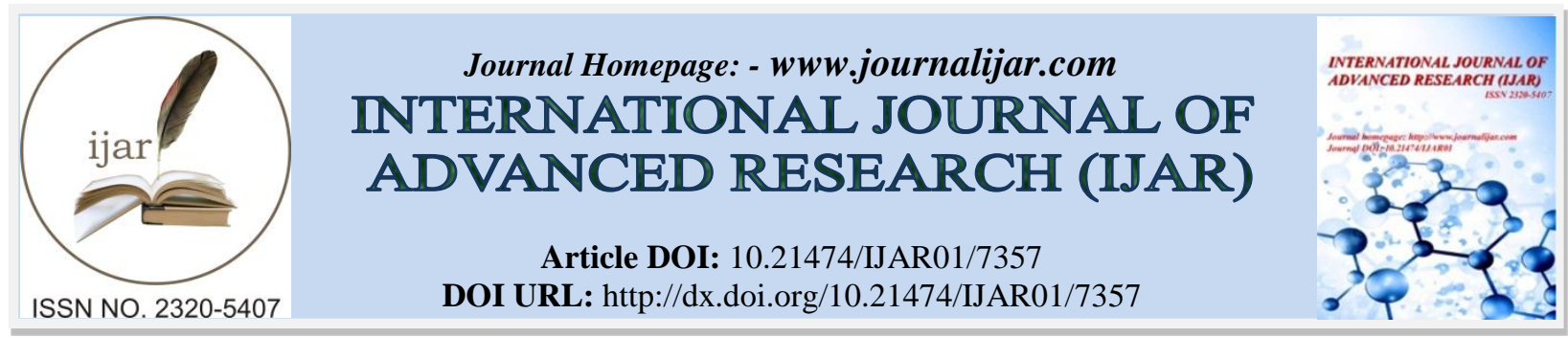

RESEARCH ARTICLE

\title{
ACTIVITY DELAY QUANTUM METHOD (ADQM).
}

\author{
Suhaib Anani. \\ 2p2c Project Management Consultants, Dubai, United Arab Emirates.
}

\section{Manuscript Info}

Manuscript History

Received: 06 May 2018

Final Accepted: 08 June 2018

Published: July 2018

Keywords:-

Construction Disputes, Delay Analysis,

Time Impact, negative float, Schedule

\begin{abstract}
The number of construction disputes are on the rise, especially that nowadays hardly any project completes on its originally agreed contractual completion date. It is noted however, that despite many advances in the ways and techniques by which delays can be assessed and ascertained, the current methods of delay analysis fall short from providing a convincing or a universally accepted technique for measuring delays and who may be held accountable for them.

Current methods of analysis highly depend on the detection of a critical path (with highest negative total float) caused by a delayed activity and which is driving the project end date. Such techniques tend to put the blame on the party that owns that delayed activity only, but neglects all other delays that have occurred but are less critical (e.g. Time Impact Analysis). Other techniques rely on the concept of selecting one or more time slices or "windows" and measuring the activities with the highest negative float within them, which again fails to consider other less critical delays in the activity network. Both the above techniques use the logic of the incomplete part of the schedule to predict the impacted end date of the project which may also be misleading as future activities are highly unpredictable.

In this paper, a new method will be introduced, that does not depend on critical paths, programme logic, or future activities to measure delays but rather an analysis technique that will measure the types of delays that every activity in the schedule has suffered to arrive at definitive results to who caused the delays and by who much.
\end{abstract}

Copy Right, IJAR, 2018,. All rights reserved.

\section{Introduction:-}

A. The need for a change in delay analysis methods

No matter how well a project was planned, or how competent the project management team is, it seems that projects are almost always destined to be delayed from its agreed contract duration. Incomplete design, unexpected subsurface conditions, and client-imposed changes are only part of what would cause major upsets to expected delivery dates of major around the globe.

As project delays are inevitable, so as the cost overruns they are likely to produce. If the Contractor believes that delays are for reasons beyond his control, he would initiate a claim requesting to recover the additional costs incurred. Over the years, owners and contractors have used various Delay Analysis Techniques to investigate the

Corresponding Author:- Suhaib Anani.

Address:- 2p2c Project Management Consultants, Dubai, United Arab Emirates. 
causes of delays. However, in the vast majority of cases, the parties are not able to settle delay claims amicably resulting in costly disputes after project completion. [3]

There are several methods by which a delay analysis for a project can be performed. The society of construction law has published a document named "the delay and disruption protocol" which provides guidelines to deal with delay and disruption issues. The latest version of the protocol issued in 2017 identifies 6 types of delay analysis that can be used in various project situations. [4]

The purpose of this research is to introduce a new method by which any and every delay occurred can be assessed (activity by activity) for both parties, while reducing or eliminating the arguments related to concurrency, incorrect logic, or misrepresentation of the dominant delay events. It is also foreseen that ADQM will present a balanced allocation of each parties liabilities as opposed to making a judgment based on the Event driving the completion date (Dominant Event).

\section{B. The incomprehensibility of the current delay methods}

The methods delay analysis described in the above section are generally categorized as using one of the following approaches:

- Time impacted programmes which identifies the cause and effect for selected delay events, then analyzing the delays to determine the event with the negative float (longest path causing delays)

- Window based analysis that considers the amount of delay for the activity with maximum delays (negative floats) during each window. Delays are then cumulated across all the windows to produce the overall delay in the project.

Both the above approaches consider the activity with the highest effect on the schedule or window (the driving activity) to be the factor that has eventually caused the overall delay to the project. The culpable party in this case, is the party that has caused the driving activity to be delayed. Other delayed activities in the schedule, who have less or no negative floats, are essentially left out in the process.

The situation becomes more difficult if concurrency of delays is to be considered in the analysis. The way by which a delay can be considered to be concurrent is not clear. One definition suggests that "a period of project over-run which is caused by two or more effective causes of delay which are of equal causative potency." [1]

The Malmaison's Approach suggests that when concurrent delay occurs, one an owner risk event and the other a contractor risk event, only one of the delays is the dominant cause for the delay to the project and prevails over the other cause of delay. [2]. Such approach is described by the Editor of Keating as "the now excepted approach in resolving concurrency in the context of extension of time claims" [5]

Another factor to consider is that total float values used to measure delays are a product of programme logic. Hence, delay analysis results are highly reliant on how activities are related (linked) in the schedule. Any missing dependencies between activities may result in an activity having a higher total float than it should be and visa versa for activities that are illogically linked to unrelated successors. Consequently, any inappropriate logic may result in changing the driving activity and hence a different outcome of the delay analysis using them.

It should be always remembered that project schedules attempt to model the activities and the way they inter-related on site. However, in an actual construction site, activities tend to be change their dependencies to become more loosely or in some cases disconnected from their dependencies. Moreover, some activities - who are not originally related - become related to others midway of their progress due to the ever-changing site conditions.

It follows from the aforesaid that using the float of driving activity to determine delays and accountability is an approach that is susceptible to errors and overlooks all the other slippages or delays that have occurred for other activities with lesser negative float. Hence, it is more likely that one party will get away with his delays simply by because the critical float path of the delays of the other party is slightly longer. The activity delay quantum method (ADQM) attempts to estimate the delays of each party in a wholistic approach, by considering the delays of both parties and for all delayed activities in the schedule. 


\section{Method Criteria:}

The ADQM method uses parameters that can be extracted from a project schedule with as built data to measure delays. The delay contribution of each activity is to be measured using a combination of the following parameters:

1. Actual Start versus the planned start (Start variance or slippage)

2. Actual duration versus the original (planned) duration

3. Float available (or proximity to longest path).

4. Weightage - Man hours (or cost)

The first 2 parameters are indicative of the amount of delay an activity has experienced during the course of its execution. Whereas the last two parameters shall be used as weight factors that will affect the activity contribution to delay, dependent on its degree of criticality (proximity to the critical path) and the material weight expressed in Manhours (or cost). Activities close to critical path or critical activities with a high value of budgeted man hours (or cost) shall have a high contribution to the delays (high impact).

\section{Start Variance Factor (SV Factor)}

If an activity is delayed from its planned early start, it is said to have a start variance (or slippage) equal to the difference between the planned start and actual start dates of an activity. Thus, start variance (SV) can be calculated as follows:

SV = Planned Start - Actual Start

A negative SV value indicates that an activity has started later than its planned start date.

To measure the effect of each activity's actual start on the overall schedule's performance, the SV factor calculates the ratio of the amount of slippage of an activity to the total float available hence:

$$
\text { SV Factor }=\frac{-(\mathrm{SV})-\mathrm{TF}(\text { Baseline })}{\mathrm{TF}(\text { Baseline })} .
$$

A high value of SV factor will indicate that the activity has been delayed for a large amount of time compared to the total float available for it, and hence had a high contribution to the overall delays in the project.

\section{Duration Variance Factor (DV Factor)}

The DV Factor measures the actual total duration of an activity compared to its original duration. For completed activities, the total actual duration is the "actual finish date" - "actual start date" for completed activities, and for activities in progress, the actual duration is measured by adding the actual duration (to date) to the remaining duration (anticipated). The DV factor is established by subtracting the original duration from the actual duration, then dividing the result by the Original duration.

DV Factor $=\quad \underline{\text { Actual Duration }- \text { Original Duration }}$

Original Duration

In essence, the DV factor is a measure of the percent deviation of the actual duration from the original duration of an activity. A greater DV value indicates that the actual duration has highly deviated from the original duration assumed at the start of the project. The larger the DV value of an activity the greater it is likely to affect the overall project performance.

\section{Total float Factor (TF Factor)}

The float will be a measure of criticality and hence, the more an activity is near critical, the more it shall affect the project in case it is delayed. Hence, the TF factor provides a weightage system whereby the near critical activities (the one with low float values or near zero) will have a higher factor than activities with high positive floats. Since, the total float has an inverse correlation with criticality, the best way to resemble the TF factor is to use the its mathmatical inverse, hence:

TF Factor $=$

$\frac{1}{\text { Total Float }}$ Where the Total Float is not zero


Note that the total floats from the updated schedule are not used in the analysis as they are affected by the progress and delays of other actvities, some of which may not belong to the same culpable party (party that caused the delay).

For activities lying on the critical path (zero float activities), the TF factor shall be considered to have a value of " 1 ". So mathematically speaking the Factor range value is $0<$ TF Factor $<=1$

\section{Weightage (Budgeted) Factor}

The weightage factor will differentiate the important (or heavy) activities from the light (or less effective) activities. This is usually achieved by either using the lumpsum cost or total manhours that are loaded on each activity in the schedule. The cost or manhours of one activity are then divided by the total cost or manhours in the project to produce the weight factor of that activity.

\section{Implementation Methodology of ADQM}

To test the feasibility of ADQM as an alternative method of time delay analysis, the method was tested using real life projects that are either completed or in progress. The projects selected for testing are the ones were an established baseline schedule and schedule updates are available using Primavera P6 project management software.

The process starts by assigning the baseline schedule to the updated schedule where the delay analysis by ADQM is to be performed. The updated schedule can be selected at any stage of the project or if the project is completed, the as built schedule (all activities updated to $100 \%$ complete) is to be used. The cutoff date (or data date) for the selected updated schedule is the date up to which delays are to be assessed.

Using Primavera P6 on the update with its baseline, a layout similar to the extracted image in Figure 1 can be produced that will show data for Original, Actual and Remaining Durations, \% complete, Baseline and Updated start and finish dates, Baseline total float, Start variance and Budgeted Units.

In order to perform further analysis on the schedule, data all the activities and their data are copied from the above layout on to an Excel spreadsheet. Then values for DV factor, TF factor, and Weightage factors are calculated from the data from P6 using the spreadsheet functions. See figure $2 \mathrm{a}, 2 \mathrm{~b}$

Fig. 1: Programme layout on Primavera P6

\begin{tabular}{|c|c|c|c|c|c|c|c|c|c|c|c|c|c|c|}
\hline \multirow{2}{*}{ 광 } & \multicolumn{14}{|l|}{ Activities } \\
\hline & \multicolumn{14}{|c|}{ Projects Activities } \\
\hline 年 & \multicolumn{14}{|c|}{$\checkmark$ Layout: AQM Analysis } \\
\hline 4 & Activity ID & $\nabla$ & Activity Name & \begin{tabular}{|c|} 
Original \\
Duration
\end{tabular} & $\begin{array}{r}\text { Actual } \\
\text { Duration }\end{array}$ & $\begin{array}{r}\text { Remaining } \\
\text { Duration }\end{array}$ & \% Comp. & BL Start & BL Finish & Start & Finish & $\begin{array}{r}\text { BL Total } \\
\text { Float }\end{array}$ & $\begin{array}{l}\text { Start } \\
\text { Var }\end{array}$ & $\begin{array}{l}\text { Budgeted Labor } \\
\text { Unts }\end{array}$ \\
\hline$\square$ & 是店 & SANITARY SE & WERAGE & 66 & 39 & 55 & & 01 Jun-15 & 20-Aug-15 & 01:Aug-15, & 19:Nov-15 & & .50 & 18380 \\
\hline 2 & $\varpi$ & A13150 & EXCAVATION TRENCHES FOR SEWERAGE PIPES COMPL & 20 & 39 & 7 & $65 \%$ & 01 Jun-15 & 23.Jun-15 & 01.Aug-15, & 22.Sep-15 & 6 & .50 & 544 \\
\hline 同 & \pm & A13160 & SUPPLY AND LAY OF SEWERAGE PIPES COMPLETE SEC & 20 & 37 & 8 & $60 \%$ & 07 Jun 15 & 29Jun 15 & 03.Aug-15, & 23.Sep.15 & 7 & -47 & 1996 \\
\hline 난 & \pm & A13165 & HTDROSTATIC TEST & 1 & 34 & 1 & $40 \%$ & 30Jun-15 & 30Jun 15 & 06-Aug-15, & $15-S e p-15$ & 11 & .30 & 30 \\
\hline 圆 & \pm & A13170 & PCC LAYING AND INSTALL MANHOLES COMPLETE SECTI & 20 & 34 & 12 & $40 \%$ & 15Jun-15 & 07.Jul-15 & 06-Aug-15, & 30-Sep-15 & 7 & .43 & 6400 \\
\hline$\cdot$ & $\Xi$ & A13480 & WARNNG TAPE \& BACKFILLING & 2 & 0 & 2 & $0 \%$ & 08 Jul-15 & 09.Jul-15 & $01 \cdot 0 \mathrm{ct} \cdot 15$ & $03 \cdot 0 \mathrm{ct} \cdot 15$ & 64 & .69 & 20 \\
\hline 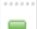 & 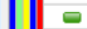 & A17120 & EXCAVATION TRENCHES FOR SEWERAGE PIPES COMPL & 20 & 0 & 20 & $0 \%$ & 24Jun-15 & 16.Jul-15 & 23-Sep-15 & $18 \cdot 0 \mathrm{ct} \cdot 15$ & 6 & .76 & 544 \\
\hline 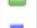 & ص & $A 17130$ & SUPPLY AND LAY OF SEWERAGE PIPES COMPLETE SEC & 20 & 0 & 20 & $0 \%$ & 30Jun-15 & 25Jul-15 & $01 \cdot 0 \mathrm{ct} \cdot 15$ & 24.0ct-15 & 32 & .76 & 1996 \\
\hline E & $\Xi$ & A17135 & HYDROSTATIC PRESSURE TEST & 1 & 0 & 1 & $0 \%$ & 26.Jul-15 & 26.Jul-15 & $25 \cdot 0 \mathrm{ct} \cdot 15$ & $25 \cdot 0 \mathrm{ct} \cdot 15$ & 32 & .76 & 30 \\
\hline . & 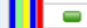 & A17140 & PCC LAYING AND INSTALL MANHOLES COMPLETE SECTI & 20 & 0 & 20 & $0 \%$ & 27 Jul-15 & 18-Aug-15 & $26 \cdot 0 c t \cdot 15$ & 17:Nov-15 & 32 & .76 & 6800 \\
\hline- & $\Xi$ & A19630 & WARNING TAPE \& BACKFILLING & 2 & 0 & 2 & $0 \%$ & 19.Aug.15 & 20-Aug-15 & 18-Nov-15 & 19-Nov-15 & 32 & .76 & 20 \\
\hline 田 & 直 & STORM DRAI & NAGE & 52 & 0 & 52 & & $25 \sqrt{\text { Jun }}-15$ & 05:Aug:15 & 24.Sep-15 & 26-Nov-15 & & .76 & 7659 \\
\hline (in & $\square$ & A13180 & EXCAVATION TRENCHES COMPLETE & 20 & 0 & 20 & $0 \%$ & 25 Jun 15 & 18 Jul-15 & $24 \cdot \mathrm{Sep} \cdot 15$ & $19 \cdot 0 \mathrm{ct} \cdot 15$ & 6 & .76 & 578 \\
\hline 8 & $\Xi$ & A13190 & SUPPLY AND LAY OF SW PIPE COMPLETE & 20 & 0 & 20 & $0 \%$ & 29.Jun-15 & 23Jul-15 & 19.0ct:15 & 11:Nov-15 & 79 & .92 & 2541 \\
\hline & $\Xi$ & A13195 & HYDROSTATIC PRESSURE TEST & 1 & 0 & 1 & $0 \%$ & 25Jul-15 & 25Jul-15 & 11-Noy-15 & 12-Nov-15 & 80 & .92 & 30 \\
\hline & e & A13200 & PCC LAYING AND INSTALL MANHOLES COMPLETE & 20 & 0 & 20 & $0 \%$ & $04 \sqrt{\text { ull}}-15$ & 28Jul-15 & $26 \cdot 0 \mathrm{ct} \cdot 15$ & 18-Nov-15 & 79 & .94 & 3540 \\
\hline & e & A13210 & GULLIES SUPPLY AND INSTALL COMPLETE & 15 & 0 & 15 & $0 \%$ & $15 \mathrm{Jul}-15$ & 03-Aug-15 & 07.Nov-15 & 24:Nov 15 & 79 & .94 & 950 \\
\hline
\end{tabular}


Fig. 2a: Sample Spread Sheet for ADQM factor Calculations -the input part

\begin{tabular}{|c|c|c|c|c|c|c|}
\hline Activity ID & Activity Name & $\begin{array}{r}\text { Original } \\
\text { Duration } \\
\square\end{array}$ & $\begin{array}{c}\text { Actual } \\
\text { Duration }\end{array}$ & $\begin{array}{c}\text { Remaining } \\
\text { Duration }\end{array}$ & \% Comp. & $\begin{array}{c}\text { Baseline } \\
\text { Total } \\
\text { Float }\end{array}$ \\
\hline A1130 & EXCAVATION TRENCHES FOR ALL SW PIPES INCLUDING BED & 26 & 54 & 0 & $100 \%$ & 2 \\
\hline A1140 & LAYING OF ALL SW PIPES COMPLETE SECTION 1 & 26 & 108 & 0 & $100 \%$ & 16 \\
\hline A1150 & HHYDROSTATIC TET & 1 & 109 & 0 & $100 \%$ & 21 \\
\hline A1290 & SUPPLY AND INSTALL OF MANHOLES COMPLETE (TYPE G) SI & 20 & 129 & 1 & $95 \%$ & 16 \\
\hline A1330 & GULLIES SUPPLY AND INSTALL AND OUTFALL SECTION 1 & 20 & 96 & 0 & $100 \%$ & 20 \\
\hline A1380 & WARNING TAPE \& BACKFILL & 2 & 97 & 0 & $100 \%$ & 28 \\
\hline A17080 & EXCAVATION TRENCHES FOR ALL SW PIPES INCLUDING BED & 26 & 55 & 0 & $100 \%$ & 2 \\
\hline A17090 & LAYING OF ALL SW PIPES COMPLETE SECTION 2 & 30 & 35 & 0 & $100 \%$ & 5 \\
\hline A17095 & HYDROSTATIC PRESSURE TEST & 1 & 22 & 0 & $100 \%$ & 5 \\
\hline A17100 & SUPPLY AND INSTALL OF MANHOLES COMPLETE (TYPE G) SI & 20 & 121 & 1 & $95 \%$ & 5 \\
\hline A17110 & GULLIES SUPPLY AND INSTALL AND OUTFALL SECTION 2 & 20 & 110 & 1 & $95 \%$ & 5 \\
\hline A19620 & WARNING TAPE \& BACKFILL & 2 & 45 & 0 & $100 \%$ & 15 \\
\hline A18770 & EXCAVATION TRENCHES FOR HDPE PIPES $500 \mathrm{~mm}$ & 12 & 77 & 11 & $10 \%$ & 15 \\
\hline A18780 & SUPPLY AND LAY OF HDPE PIPES 500mm COMPLETE & 6 & 75 & 5 & $10 \%$ & 15 \\
\hline A1890 & ELECTRICAL WORKS AND STREET LIGHTING COMPLETE SUPI & 15 & 95 & 11 & $30 \%$ & 19 \\
\hline A1900 & UNDER GROUND DUCTS AND UTILITY STRUCTURE AND TEL & 7 & 93 & 6 & $20 \%$ & 19 \\
\hline A1910 & UNCLASSIFIED HIGHWAY EXCAVATION & 10 & 37 & 0 & $100 \%$ & 15 \\
\hline A1920 & FILL & 15 & 79 & 0 & $100 \%$ & 15 \\
\hline A1930 & SUPPLY AND LAY SUB BASE LAYER & 10 & 79 & 0 & $100 \%$ & 15 \\
\hline A1940 & SUPPLY AND LAY ROAD BASE LAYER & 15 & 61 & 2 & $90 \%$ & 15 \\
\hline A1950 & SUPPLY AND FIX NON-MOUNTABLE CURB & 15 & 60 & 2 & $90 \%$ & 15 \\
\hline
\end{tabular}

Fig. 2b: Sample Spread Sheet for ADQM factor Calculations - the output part

\begin{tabular}{|c|c|c|c|c|c|c|c|c|c|}
\hline Activity ID & $\begin{array}{l}\text { Start Var } \\
\text { Factor }\end{array}$ & $\begin{array}{l}\text { Duration } \\
\text { Variance }\end{array}$ & DV Factor & $\begin{array}{c}\text { TF } \\
\text { Factor }\end{array}$ & $\begin{array}{l}\text { Weightage } \\
\text { Factor }\end{array}$ & $\begin{array}{c}\text { ADQM } \\
\text { Factor (A) }\end{array}$ & $\begin{array}{c}\text { ADQM } \\
\text { Factor (B) }\end{array}$ & $\begin{array}{l}\text { Culpability } \\
\text { (A) }\end{array}$ & $\begin{array}{l}\text { Culpability } \\
\text { (B) }\end{array}$ \\
\hline$\sigma$ & $\checkmark$ & $\nabla$ & 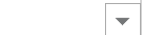 & $\sigma$ & 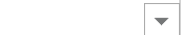 & 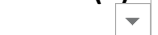 & 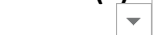 & $\checkmark$ & - \\
\hline A1130 & 16 & 28 & 1.076923 & 0.5 & 0.00016977 & 0.0013582 & $9.141 \mathrm{E}-05$ & 1 & 2 \\
\hline A1140 & 1.125 & 82 & 3.153846 & 0.063 & 0.000930019 & $6.539 \mathrm{E}-05$ & 0.0001833 & 1 & 2 \\
\hline A1150 & 0.380952 & 108 & 108 & 0.048 & $9.28428 \mathrm{E}-06$ & $1.684 \mathrm{E}-07$ & 4.775E-05 & 1 & 2 \\
\hline A1290 & 2.25 & 110 & 5.5 & 0.063 & 0.004376873 & 0.0006155 & 0.0015045 & 1 & 2 \\
\hline A1330 & 2.4 & 76 & 3.8 & 0.05 & 0.003660657 & 0.0004393 & 0.0006955 & 1 & 2 \\
\hline A1380 & -0.035714 & 95 & 47.5 & 0.036 & 7.95795E-06 & $-1.015 \mathrm{E}-08$ & 1.35E-05 & 1 & 2 \\
\hline A17080 & 11 & 29 & 1.115385 & 0.5 & 0.00016977 & 0.0009337 & $9.468 \mathrm{E}-05$ & 1 & 2 \\
\hline A17090 & 7.6 & 5 & 0.166667 & 0.2 & 0.00076025 & 0.0011556 & $2.534 \mathrm{E}-05$ & 1 & 2 \\
\hline A17095 & 6.4 & 21 & 21 & 0.2 & 1.19369E-05 & $1.528 \mathrm{E}-05$ & $5.014 \mathrm{E}-05$ & 1 & 2 \\
\hline A17100 & 8 & 102 & 5.1 & 0.2 & 0.002191089 & 0.0035057 & 0.0022349 & 1 & 2 \\
\hline A17110 & 9.6 & 91 & 4.55 & 0.2 & 0.001538537 & 0.002954 & 0.0014001 & 1 & 2 \\
\hline A19620 & 2.466667 & 43 & 21.5 & 0.067 & 7.95795E-06 & $1.309 \mathrm{E}-06$ & $1.141 \mathrm{E}-05$ & 1 & 2 \\
\hline A18770 & 3.4 & 76 & 6.333333 & 0.067 & 7.79879E-05 & $1.768 \mathrm{E}-05$ & $3.293 E-05$ & 2 & 2 \\
\hline A18780 & 3.266667 & 74 & 12.33333 & 0.067 & 0.000448828 & $9.774 \mathrm{E}-05$ & 0.000369 & 2 & 2 \\
\hline A1890 & 1.368421 & 91 & 6.066667 & 0.053 & 0.000225475 & $1.624 \mathrm{E}-05$ & 7.199E-05 & 2 & 2 \\
\hline A1900 & 0.631579 & 92 & 13.14286 & 0.053 & 0.000326276 & $1.085 \mathrm{E}-05$ & 0.0002257 & 1 & 2 \\
\hline A1910 & -1.666667 & 27 & 2.7 & 0.067 & 8.011E-05 & $-8.901 E-06$ & $1.442 \mathrm{E}-05$ & 2 & 2 \\
\hline A1920 & 1.333333 & 64 & 4.266667 & 0.067 & 0.000517267 & $4.598 \mathrm{E}-05$ & 0.0001471 & 2 & 2 \\
\hline A1930 & 1.333333 & 69 & 6.9 & 0.067 & 0.000919939 & $8.177 \mathrm{E}-05$ & 0.0004232 & 2 & 2 \\
\hline A1940 & 3.4 & 48 & 3.2 & 0.067 & 0.000976175 & 0.0002213 & 0.0002083 & 2 & 2 \\
\hline
\end{tabular}


Fig. 4: Analysis results for SV and DV factors
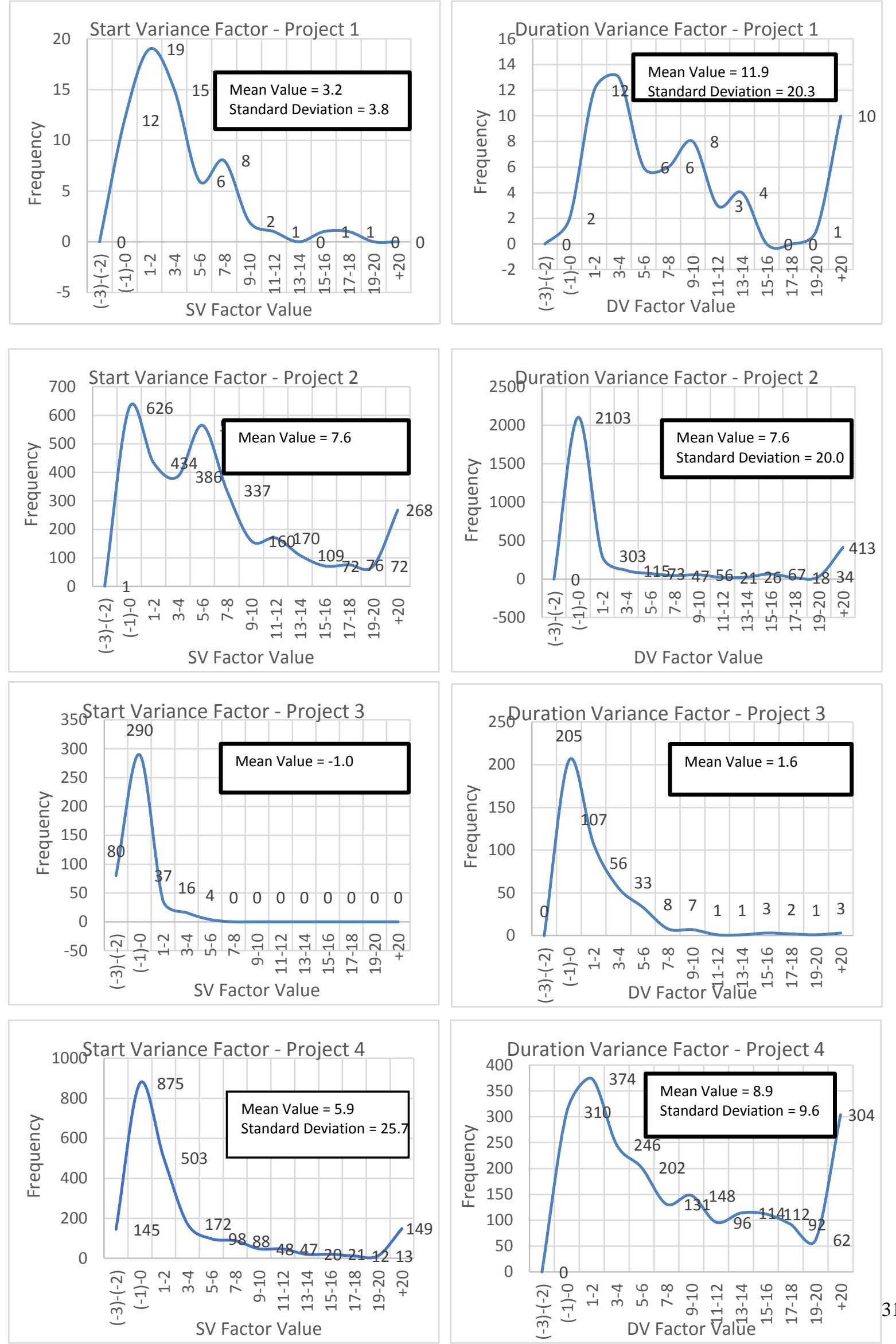


\section{The ADQM factors}

To combine the effect of individual activity delay factors, the ADQM factors are the product of SV, DV, TF and weightage factors as follows:

\section{ADQM Factor-A = (SV Factor) X (TF Factor) X Weightage Factor \\ $\mathrm{ADQM}$ Factor-B $=(\mathrm{DV}$ Factor $) \mathrm{X}$ (TF Factor) $\mathrm{X}$ Weightage Factor}

The above multiplication provides a two stage weight factorization for each of the factors involved. The resultant value of ADQM factors represents the amount of contribution such activity had to the overall delay in the project.

Fig. 3: ADQM factorization process

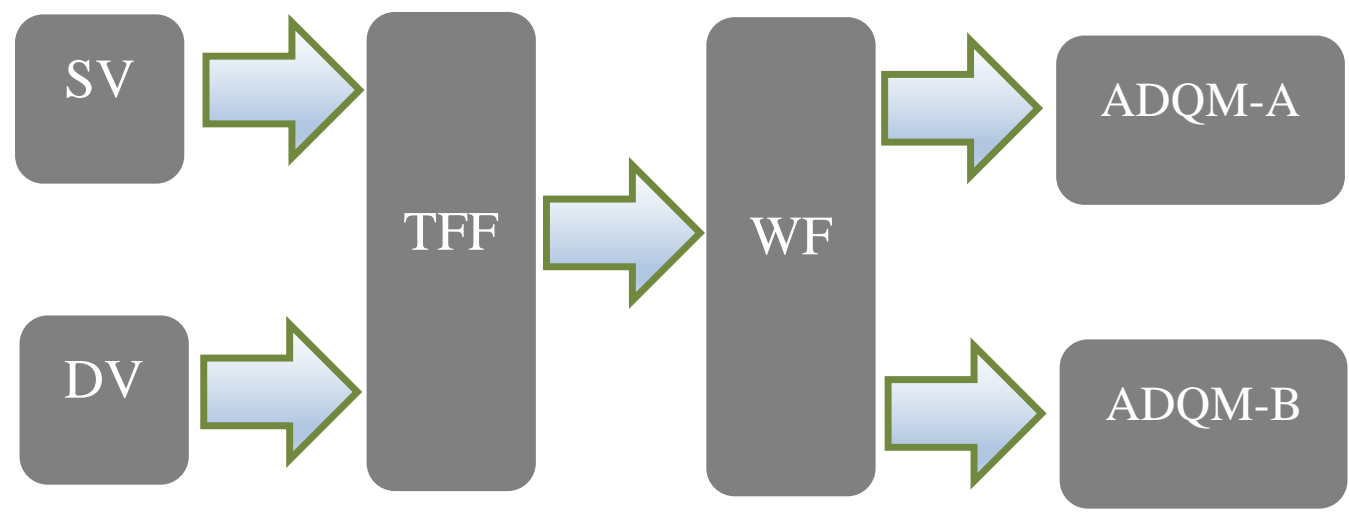

Where ADQM-A represents the resultant delay factor due to SV, while ADQM-B is the resultant delay factor due to the duration variance DV.

It is important to note that activities with zero durations, activities that have no budgeted quantities, and activities with $0 \%$ complete are all disregarded from the analysis, and are to be filtered out from the spreadsheet table.

The final step is to identify the party that is responsible for the delay of each activity. This step is critical to the ADQM results as it will define the contribution of each party to the overall delay of the project. In the last 2 columns in the table in figure 2 above, the value of 1 means that this activity was delayed by the Employer, whereas a value of 2 means that the delay was caused by the Contractor. The total ADQM factor value of each party represents its overall contribution to the delay.

\section{ADQM Field Implementation}

Preference was given to projects that were completed or expected to be completed within 2 years after their contract completion date. 4 projects with different sizes and scope were selected were an approved baseline with as built schedules exist. The following are the main characteristics of each project:

Table 1:- Characteristics of selected projects

\begin{tabular}{|l|l|l|l|l|}
\hline Project & Type & $\begin{array}{l}\text { Contract } \\
\text { Duration } \\
\text { (Months) }\end{array}$ & $\begin{array}{l}\text { Delay } \\
\text { (Months) }\end{array}$ & Size \\
\hline Project 1 & Infrastructure & 14 & 15 & Medium \\
\hline Project 2 & Villa complex & 14 & 21 & Large \\
\hline Project 3 & Medium Rise Apartment Building & 20 & 4 & Medium \\
\hline Project 4 & Mall / Retail complex & 24 & 14 & Large \\
\hline
\end{tabular}

Then the history of delays that have incurred during project execution was obtained to get a better understanding of what has caused such delays and who is the culpable party in each instance. In some instances, it was not clear why some activities were delayed as there was no obvious cause. Such delays are usually attributed to the Contractor. 
It was noted that for all projects selected, the reasons for delay were typically related to insufficient design or design changes, non-availability of Authority approvals, insufficient manpower on site, and late procurement of materials.

Fig. 5: SV Factor (Mean and SD values) - All projects

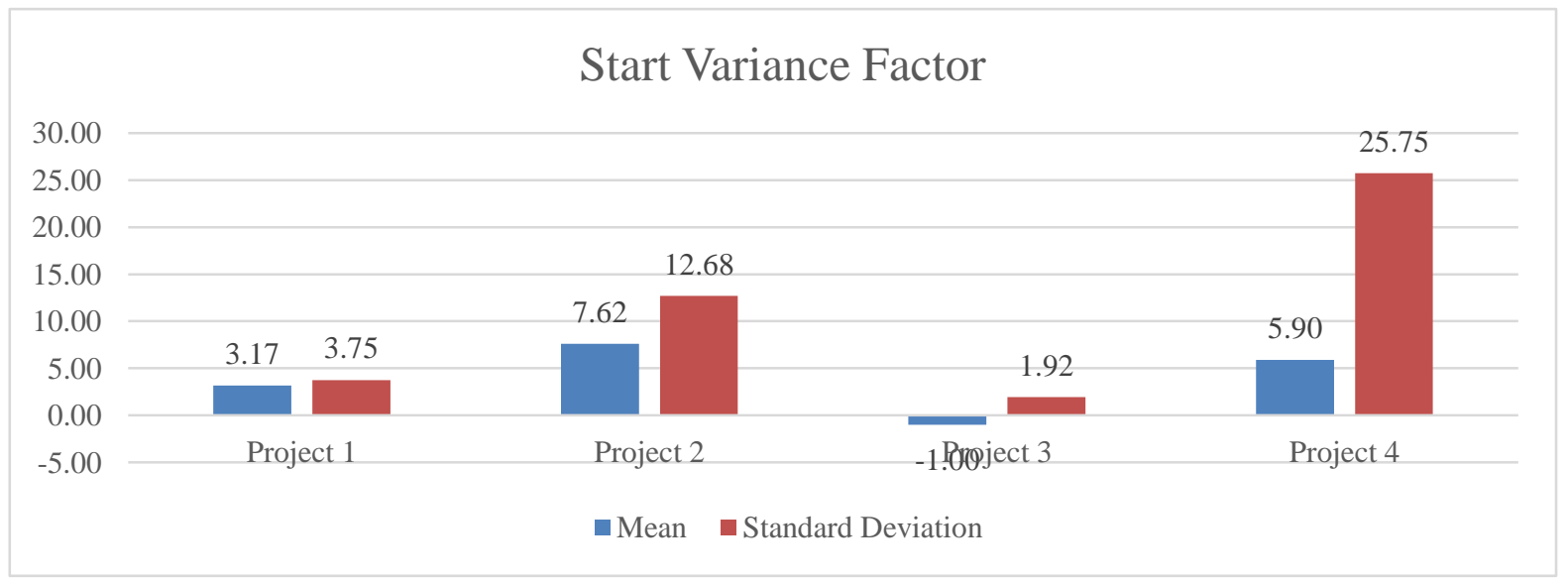

Fig. 6: DV Factor (Mean and SD values) - All projects

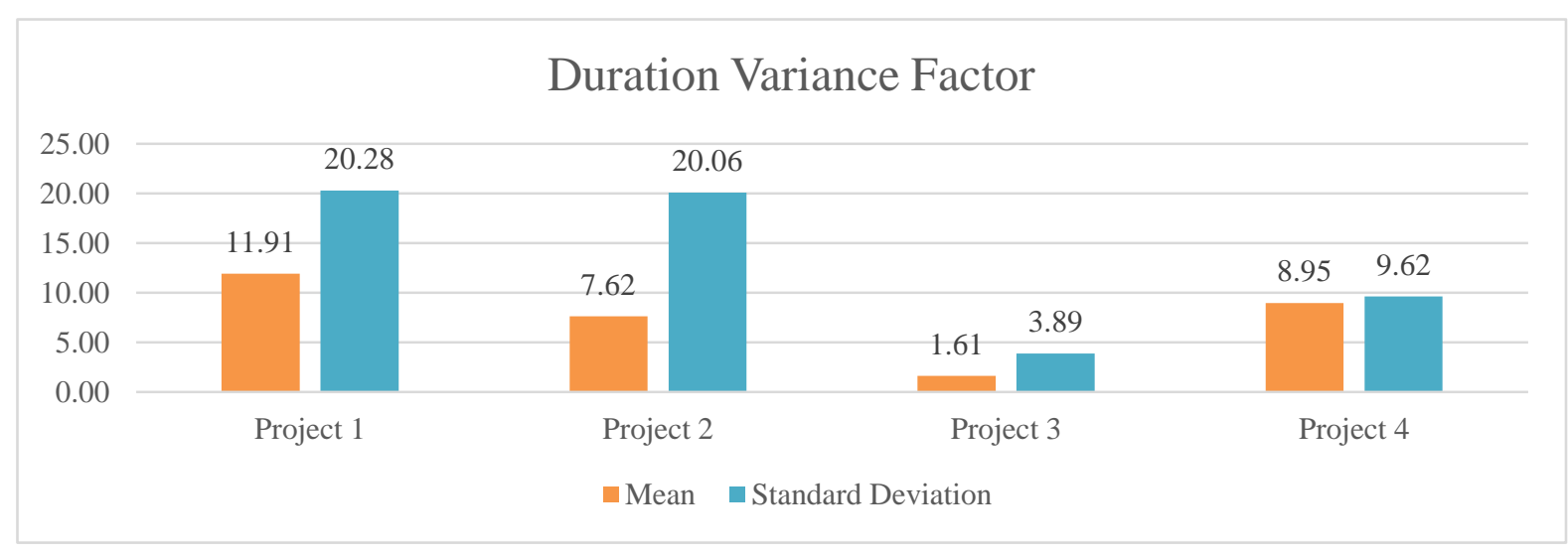

Using the layout in figure 1, the data in the P6 table was copied into the excel spreadsheet for each project. Using the formulas in the spreadsheet the values of SV, DV, and ADQM factors were established for each activity. Figure 4 shows the results for SV and DV factors.

The SV factor can be thought of as a ratio between the number of days an activity was late from its early start to the total float available to that activity. If the ratio is larger than 1, then it can be assumed that had the float of the activity be available to it, the activity would have consumed an excess of that float.

Comparing the results of the 4 projects in figure 4 , it can be said (except for project 3 ) that the Delays from late started activities can, in average, consume as much as 7 times more that the total float available to them. Which means that the parties are unaware of the true volume of delays that have occurred in any given project due to their failure to start activities when they were supposed to be started. It is also observed that the SV factor curves always peak at 0-2 value which shows that the majority of activities do not exceed double the total float available to them. Similar findings are resonated for the curves for the DV factors.

Figures 5 and 6 show the Mean and standard deviation values for Both SV and DV factors across the 4 projects. When compared to each other, the Mean values show that the delays caused by activity late starts (SV) are much less than the delays caused due to extending activity duration (DV). Data collected from the sample projects show that the effect of Duration variances can be 3 times more than that of start variances. 
Fig. 6: DV Factor (Mean and SD values) - All projects

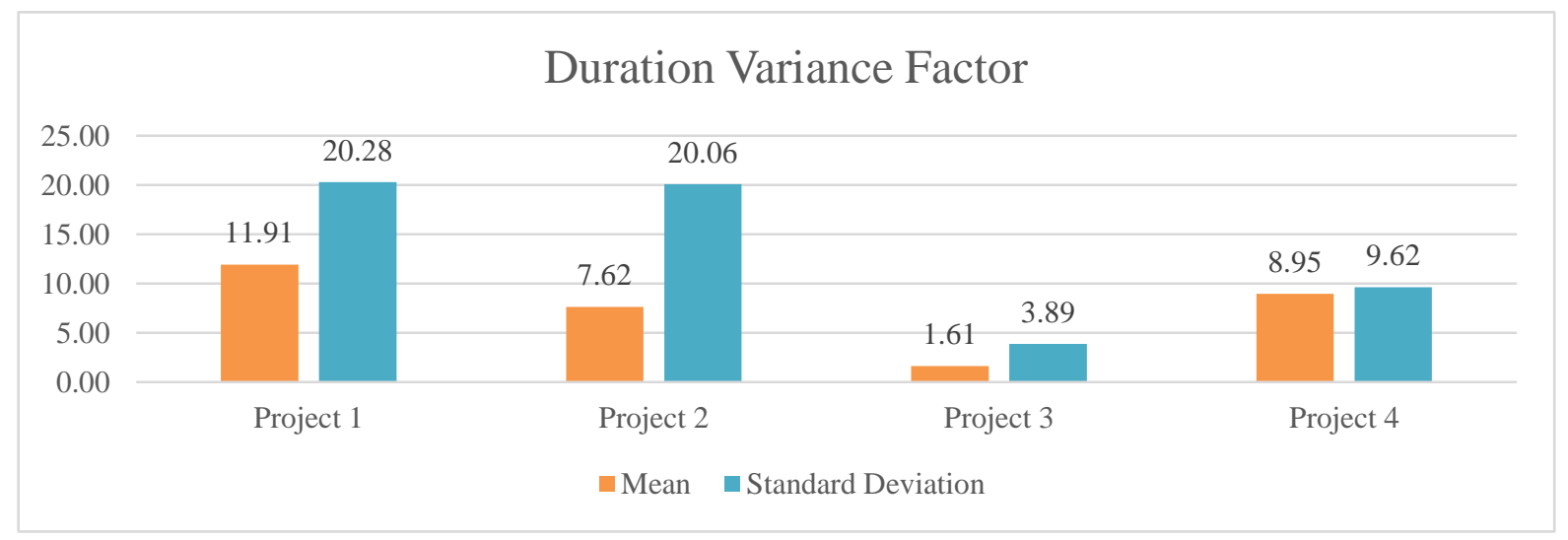

Standard deviation values are an indication of how close or scattered the SV or DV factor values are from the Mean value. For example, in project 4, the standard deviation value for SV Factor is 25.75 which may be an indication that the several activities have far exceeded the total float limits when their SV values are considered, and that the responsible parties were not able to control the project's progress to minimize the late activity starts.

The low SV and DV factor values for project 3 are characteristic for project with good performance and low delays. As project 3 was in progress when the data was collected for analysis, it is anticipated that the factor values will increase especially if the project experience delays towards its end date.

As previously discussed, the output of the last 2 columns in figure 2 is used to determine how much each party has contributed to the delays. ADQM-A and ADQM-B are the two parameters that calculate the weighted effect of the SV and DV factors respectively. The contribution of the Employer is the sum of its part in both ADQM values hence:

Employer contribution $(\mathrm{EC})=\mathrm{ADQM}-\mathrm{A}($ Employer $)+\mathrm{ADQM}-\mathrm{B}($ Employer $)$

Similarly,

Contractor contribution $(\mathrm{CC})=\mathrm{ADQM}-\mathrm{A}($ Contractor $)+\mathrm{ADQM}-\mathrm{B}$ (Contractor)

And the \% contribution of both the Employer and the Contractor is:

Employer \% contribution $=\frac{\mathrm{EC}}{\mathrm{EC}+\mathrm{CC}} \%$

Contractor $\%$ contribution $=\frac{\mathrm{CC}}{\mathrm{EC}+\mathrm{CC}} \%$ 
Fig. 7: Parties Contribution to delays using the ADQM

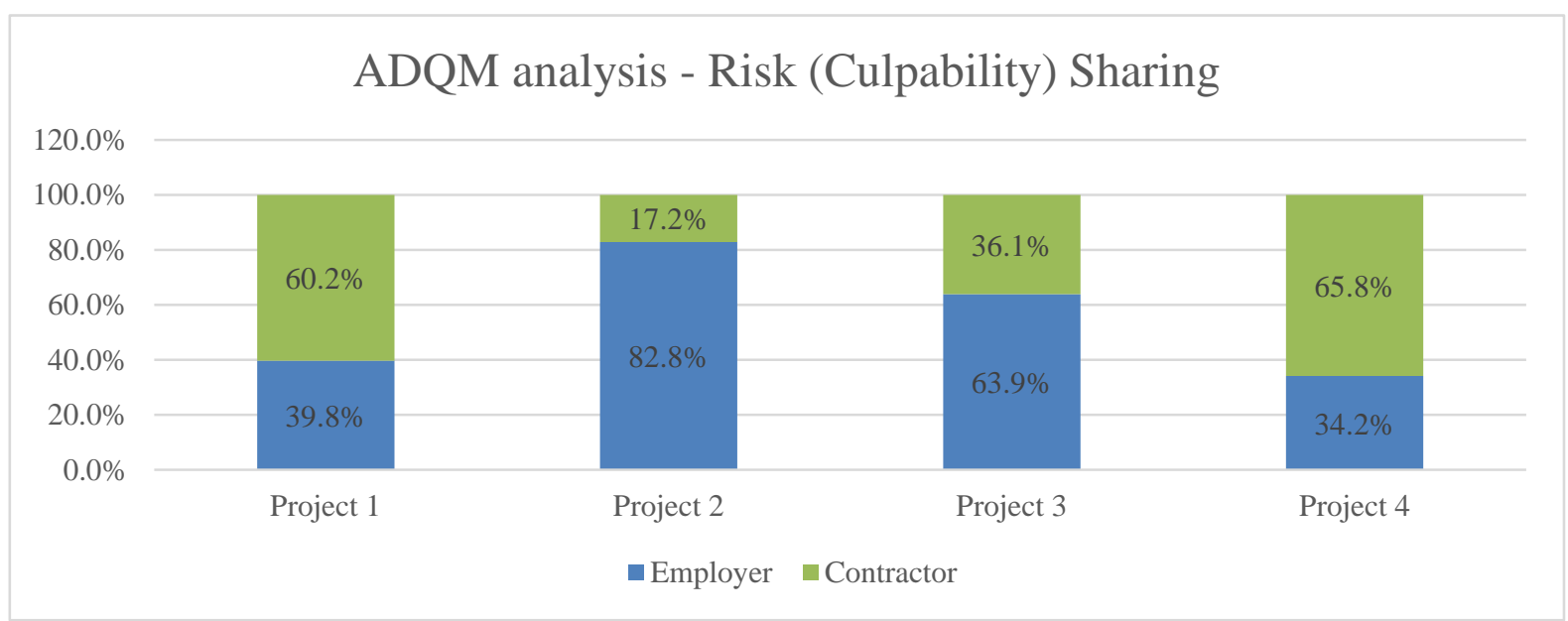

Figure 7 shows the results of the application of ADQM analysis. It is noted that ADQM has demonstrated that for all the projects considered, the responsibility for delays is shared between the parties. Results also show that it is more likely than not that the Contactor is a major contributor to the delays in the project, and that any party is likely to contribute to at least one third of the delays encountered. This is contrary to the current practices that suggest that one party is usually the sole culprit of delays.

The final step is to multiply the \% share of each party by the total delay in the project which is obtained from the updated schedule to obtain the delay in days caused by each party. The same \% share can be multiplied by the total additional costs caused by the delay to determine the cost share of each party.

\section{Conclusion:-}

Current methods of analysis are primarily used to analyze a limited number of isolated delay events from which an event with the highest impact would be considered as the main cause. This approach points the figure to the party being solely responsible for the delays and the consequences thereof. Even when a window analysis is performed, each window will still select the activity with the highest impact in that window leaving other delays undetected. Hence, it can be said that the current methods of time delay analysis are unable to provide a fair and reasonable way of allocating delays to their responsible parties.

The introduction of ADQM attempts to provide a holistic approach were any delay (whether critical or not) is deemed to have a contribution to the overall delay of the project. It is anticipated that if ADQM is used correctly, it would result in a considerable reduction in the number of disputes that are currently experienced around the world.

The results of ADQM parameters can also be used as key performance indicators so as to understand the reasons for delay and explore ways to reduce or avoid them.

\section{References:-}

1. Concurrent Delay, John Marrin QC, February 2002. A paper given at a meeting of the Society of Construction Law on February $5^{\text {th }}, 2002$.

2. Analysis of Concurrent Delays on Construction Claims, Richard J. Long, P.E., Long International

3. Construction Delay Analysis Techniques-A Review of Application Issues and Improvement Needs, Nuhu Braimah, Buildings 2013, 3, 506-531; doi:10.3390/buildings3030506

4. Society of Construction Law, Delay and Disruption Protocol, $2^{\text {nd }}$ Edition, February 2017.

5. "Keating on Construction Contracts," 9111 Edition, Sweet \& Maxwell, 2012, paras. 9-062-9-066 